\title{
Topical Issue on atomic cluster collisions ${ }^{\star}$
}

\author{
Alexey Verkhovtsev ${ }^{1,2, a, c}$, Germán Rojas-Lorenzo ${ }^{3}$, Jesús Rubayo-Soneira ${ }^{3}$, and Andrey V. Solov'yov,b,c \\ 1 MBN Research Center, Altenhöferallee 3, 60438 Frankfurt am Main, Germany \\ 2 Department of Medical Physics in Radiation Oncology, German Cancer Research Center, Im Neuenheimer Feld 280, 69120 \\ Heidelberg, Germany \\ 3 Instituto Superior de Tecnologías y Ciencias Aplicadas (InSTEC), Havana University, AP 6163 La Habana, Cuba
}

Received 23 May 2018

Published online 25 July 2019

(c) EDP Sciences / Società Italiana di Fisica / Springer-Verlag GmbH Germany, part of Springer Nature, 2019

\begin{abstract}
The rapidly emerging field of atomic cluster collisions attracts strong research interest of many experimental and theoretical groups worldwide. Being a highly interdisciplinary field, it has numerous links with atomic, molecular and optical physics, astrophysics, plasma physics, biophysics, physical chemistry, solid state physics and even molecular biology. This Topical Issue presents a state-of-the-art description of current research activities in the field of atomic cluster collisions and atomic cluster science. The contributions to this Issue represent experimental, theoretical and computational studies both at the fundamental level of elementary mechanisms and at a more applied level which is necessary in numerous applications of nano- and biotechnology, materials science and medicine.
\end{abstract}

\section{Introduction}

Recent years have witnessed extensive development of experimental, theoretical and computational methods for the analysis of structure and dynamics of atomic, molecular and biomolecular cluster systems of various degree of complexity $[1,2]$. This enabled studying dynamical phenomena and many-body effects taking place in atomic clusters and nanoparticles, nanostructures, molecular and biological systems, being in the gas phase, deposited on surfaces or embedded in molecular environments. These studies have particularly focused on the problems of structure formation, stability and fragmentation [3-7], collective electron excitations [8-11], phase transitions [12,13], structural transformations [14,15], and many others. It was recognized, both experimentally and theoretically, that atomic clusters and complex molecular systems often possess unique properties, which make them interesting objects of research, rather different from both single atoms and the solid state.

Modern experimental techniques have made it possible to study properties of atomic clusters thus providing a better understanding of the transition from a single atom or

\footnotetext{
* Contribution to the Topical Issue "Atomic Cluster Collisions", edited by Alexey Verkhovtsev, Andrey V. Solov'yov, Germán Rojas-Lorenzo, and Jesús Rubayo Soneira.

a e-mail: verkhovtsev@mbnexplorer.com

b e-mail: solovyov@mbnresearch.com

${ }^{\mathrm{c}}$ On leave from Ioffe Institute, Politekhnicheskaya 26, 194021 St. Petersburg, Russia.
}

molecule to the solid state limit. In particular, structural and dynamical properties of cluster systems are commonly studied by means of photonic, electronic and atomic collisions (see, e.g., [1,2] and references therein). More recent approaches to study atomic clusters and complex molecular systems include also the employment of intense radiation sources such as free electron lasers [16]. Interaction of atomic clusters with intense laser pulses is relevant for studying the formation of nanoplasmas, which provide ideal conditions to temporally resolve ultrafast electronic processes relevant for large-scale plasmas [17].

In gas-phase experiments, free atomic and molecular clusters are probed with radiation beams (X-rays, electrons, ions), and interaction of radiation with the target leads to ionization and fragmentation of the latter. Electron and ion mass-spectrometry methods are used to analyze charged fragments and obtain information on electronic and geometrical structures of the targets. Novel experimental techniques for production of complex molecular (especially, biomolecular) targets in the gas phase, such as laser-induced acoustic desorption, matrixassisted laser desorption and ionization, and electrospray ionization, allow to explore irradiation-driven processes involving large macromolecules that are more relevant for technological and biomedical applications [18-20].

Understanding of the properties of clusters deposited on a surface or embedded into a medium is another research area of high technological relevance. Cluster-based materials, including supported clusters, ligand-protected clusters, and cluster composites, have gained much attention in recent years, in both basic science and practical 
applications. The knowledge of elemental composition, structure and electronic properties of such systems is essential for the fabrication of new materials and nanostructures, manufacturing of thin films and surface coatings, design of drugs and radiosensitizing agents, as well as for the understanding of fundamental problems, such as the functioning of quantum and thermodynamic laws on the nanoscale or mechanisms for the formation and self-assembly of complex many-atom systems.

The field of atomic cluster science is highly interdisciplinary and has numerous links with traditional branches of physics and chemistry. The premier conference in this field, the International Symposium on Atomic Cluster Collisions (ISACC) took place in St. Petersburg, Russia in 2003 [1]. Since then, eight ISACC conferences took place [1,2,21-23], including the most recent ISACC 2017 conference which was held in Varadero, Cuba in October $2017^{1}$. Initially ISACC was focused mainly on dynamics of atomic clusters, especially in atomic cluster collisions $[1,24]$. Since then its scope has been widened significantly to include dynamics of nanosystems, biomolecules and macromolecules with the emphasis on the similarity of clustering phenomena arising in different areas of physics, chemistry and biology $[2,21]$.

This Topical Issue presents a snapshot of the current research activities in different areas of atomic cluster science. It consists of 23 contributions [25-47] representing experimental and theoretical studies both at the fundamental level of elementary mechanisms and at the more applied level which is necessary in numerous applications of nano- and biotechnology, materials science and medicine.

Since the field of atomic cluster science is very broad and diverse, it is not possible to cover all research topics involving atomic clusters in a single volume. This Topical Issue highlights the latest achievements in the understanding of (i) structure and dynamics of atomic cluster systems, (ii) molecular-level mechanisms of radiation-induced biodamage, and (iii) mechanisms behind the radiosensitizing properties of atomic and molecular cluster systems. These and many other topics involving different types of clusters, various cluster geometries and size ranges, composite and ligand-protected clusters, complex molecular and biomolecular systems, clusters on a surface and embedded into nanodroplets or matrices, are among the key topics of the ISACC conference series.

Particular focus in this Topical Issue is made on the understanding of stability, electronic and geometrical properties of selected atomic cluster systems, fullerenes and other carbon-based nanosystems in electron- and ioncluster collisions, interaction of clusters with surfaces and laser fields. Studies of radiation and collision processes with biomolecular systems are highly relevant in connection with the molecular-level assessment of biological damage induced by radiation [48-50]. The scientific interest in obtaining a deeper understanding of radiation damage

1 The information about all previous and upcoming ISACC conferences, including conference programs and books of abstracts, can be found on a dedicated web-portal of the ISACC conference series, http://www.isacc-portal.org/ is motivated by the development of modern radiotherapy techniques such as ion-beam cancer therapy [49,51,52]. Another hot topic of atomic cluster science is related to the development of radiosensitizers - molecular or cluster systems that enhance radiation effects on biological targets $[53,54]$. These systems can be exploited in combination with radiotherapy to increase the effectiveness of treatment.

The contributions to this Issue cover such areas as spectroscopy, photodissociation, reaction dynamics of neutral and charged cluster systems, electron- and ion-induced fragmentation, radiation chemistry, radiation biology, and many others. A number of contributions was made by the participants of ISACC 2017 conference who presented their novel and original results.

\section{Structure and dynamics of atomic cluster systems}

Several papers in this Topical Issue describe recent advances in the understanding of stability, electronic and geometrical properties of selected cluster systems. Particular focus is made on the problems of electron-cluster collisions, interaction of atomic clusters with surfaces and the behaviour of clusters in laser fields.

The paper by Raggl et al. [31] reported the results of a gas-phase study of electron ionization of copperwater complexes formed in ultracold helium nanodroplets. This study was motivated by a potential use of copper nanoparticles as low-cost candidates for the catalytic splitting of water and production of hydrogen gas. Measured mass spectra show that $\mathrm{H}_{2} \mathrm{O}-\mathrm{Cu}$ complexes containing tens of copper and water molecules can be formed in the helium droplets. Although no ion signal for the clusters corresponding to the release of $\mathrm{H}_{2}$, such as $\left(\mathrm{H}_{2} \mathrm{O}\right)_{n-2}(\mathrm{OH})_{2} \mathrm{Cu}_{m}^{+}$or $\left(\mathrm{H}_{2} \mathrm{O}\right)_{n-1} \mathrm{OCu}_{m}^{+}$, was detected, intense signals for other types of products, such as $\left(\mathrm{H}_{2} \mathrm{O}\right)_{n} \mathrm{OHCu}_{2}^{+}$as well as $\mathrm{He}_{6} \mathrm{Cu}^{+}, \mathrm{He}_{12} \mathrm{Cu}^{+}$and $\mathrm{He}_{24} \mathrm{Cu}^{+}$, were observed.

The paper by Ciborowski et al. [35] demonstrated capabilities of the combination of a Rydberg electron transfer (RET) anion source and velocity map imaging-anion photoelectron spectroscopy (VMI-aPES) to form dipole bound anions and measure their photoelectron spectra. For these purposes, the dipole bound anions of acetonitrile, ammonia-water dimer, water dimer, dimethyl sulfoxide and thymine were chosen as examples.

In reference [40] the interaction of size-selected anionic tin clusters $\operatorname{Sn}_{n}^{-}(n=7-75)$ with electrons was studied experimentally. Apart from electron attachment and the formation of doubly and triply charged clusters with the size of the precursors, different fragments such as $\mathrm{Sn}_{n-7}^{-}$, $\mathrm{Sn}_{n-10}^{-}$or $\mathrm{Sn}_{n-15}^{-}$as well as $\mathrm{Sn}_{10}^{-}$and $\mathrm{Sn}_{15}^{-}$were observed. This fragmentation pattern was explained by fission of doubly charged clusters. The dissociation pathways of $\mathrm{Sn}_{n}^{-}$ resemble those of lead clusters, studied recently by the same group [55].

Experimental techniques, rapidly becoming more and more accurate, demand state-of-the-art theoretical and 
computational methodologies for interpretation of the measurements. In reference [25] a theoretical method for calculating and characterizing rovibrational spectra of small rare gas clusters was applied to the case of a neon trimer, $\mathrm{Ne}_{3}$. The authors assigned the symmetry to the rovibrational spectrum of $\mathrm{Ne}_{3}$ based on the solution of the rotational Hamiltonian by means of a purely vibrational basis combined with standard rotational functions. The calculated rovibrational energy levels were benchmarked against the results of other calculations reported in literature.

In reference [27] path integral molecular dynamics simulations were performed to study reactivity of $\mathrm{Rb}$ atoms on the surface of helium clusters ${ }^{4} \mathrm{He}_{n}$ and the formation of rubidium dimers in the triplet state. High-spin alkali dimers have attracted considerable attention due to their role in producing samples of cold molecules. Based on an accurate potential energy surface (PES) for the $\mathrm{He}-\mathrm{Rb}_{2}\left({ }^{3} \Sigma_{u}^{+}\right)$interaction, the total PES was described analytically through pairwise interactions. Helium clusters containing up to 70 atoms, both pure and doped with $\mathrm{Rb}_{2}$ molecule, were considered.

The study of excitation and dissociation dynamics of weakly-bound van der Waals complexes is another topic of interest. Two papers in this Topical Issue are devoted to the processes of energy relaxation and photofragmentation of a NO molecule and a van der Waals-type NO dimer. Photoexcitation of a NO molecule, embedded in argon, neon and parahydrogen cryogenic solids, to its first electronic Rydberg state was studied in reference [44]. Classical molecular dynamics approach was used with a quantum correction for the temperature using an anharmonic approach. Potential energy surfaces for the $\mathrm{NO}-\mathrm{Ne}$, NO-Ar and $\mathrm{NO}-\mathrm{H}_{2}$ systems were used to describe the dynamical response of the solid matrices upon NO photoexcitation. The results of simulations partially reproduced experimental results. In the case of the $\mathrm{NO}-\mathrm{Ne}$ system, the results were in good agreement with earlier works. Borrell-Grueiro et al. [30] studied photofragmentation of $(\mathrm{NO})_{2}$ employing a quasi-classical molecular dynamics approach. Initial conditions of the system were defined using quantum probabilities distributions for different intramolecular and intermolecular vibrational levels as well as using the Wigner distribution functions. It was observed that photofragmentation of $(\mathrm{NO})_{2}$ depends strongly on the excitation of the initial state.

In reference [47] a novel methodology was proposed to introduce retarded potentials in electron dynamics simulations based on real-time time-dependent densityfunctional theory. Two types of retardation were investigated; the first one deals with retardation in the potential created by a collision with a charged projectile. This was done through the Liénard-Wiechert potential. The second one deals with retardation in the electrostatic interaction between the time-dependent electron density and its environment. It was found that the latter effect can be safely neglected because of the rapid damping vs. distance of the electric fields created by electrostatic dipole moments. On the other hand, the use of the Liénard-Wiechert potential for modelling ion-molecule collisions by first-principles simulations was suggested.
Calvo et al. [32] determined stable structures of argon clusters doped with a strontium cation using a manybody polarizable potential fitted to reproduce highly accurate electronic structure calculations at the coupled cluster level of theory. The basin-hopping global optimization method was employed to define the lowest energy structures of $\mathrm{Sr}^{+} \mathrm{Ar}_{n}$ clusters with $n<160$. The results suggested an overall icosahedral growth pattern in the investigated size range, with $\mathrm{Sr}^{+}$cation being generally twelve-fold coordinated by argon atoms also arranged into an icosahedron. However, the strain between the first coordination shell and the remaining cluster was found significant, leading to the formation of non-icosahedral coordination shells with local decahedral or cubic arrangements.

Reber and Khanna [46] performed a density-functional theory (DFT) study of the electronic structure, stability and magnetic properties of a series of transition metal (TM)-chalcogenide clusters stabilized by $\mathrm{PH}_{3}$ ligands. The focus was made on $\mathrm{TM}_{6} \mathrm{X}_{8}\left(\mathrm{PH}_{3}\right)_{6}(\mathrm{TM}=\mathrm{Cr}, \mathrm{Mo}, \mathrm{Re}, \mathrm{Co}$; $\mathrm{X}=\mathrm{Se}, \mathrm{Te})$ and $\mathrm{Ni}_{6} \mathrm{X}\left(\mathrm{PH}_{3}\right)_{6}(\mathrm{X}=\mathrm{Se}, \mathrm{Te})$ clusters, which are of interest as the primitive builiding blocks for the formation of bulk materials with desirable functionalities. It was demonstrated that the $\mathrm{PH}_{3}$ ligands act as charge donors, transferring charge to the metal sites. They also have a strong effect on the ionization characteristics in that the first and second ionization energies are considerably reduced from their values for the non-ligated species.

Much attention has also been devoted to the study of stability, electronic properties as well as irradiation- and collision-induced processes involving carbon fullerenes. Small carbon clusters also attracted attention of physicists and chemists as they play a key role in the chemistry of carbon-rich stars, comets and interstellar molecular clouds, while acting as building blocks in the formation of complex carbon-containing compounds.

Advanced ab initio calculations of stability of smallsize carbon clusters were reported in reference [33] of this Topical Issue. A wave function-based approach was employed based on the second-order Møller-Plesset perturbation calculations with extrapolation of the energy from the lowest steps of the hierarchical staircase to the complete basis set limit. It was found that such a wave function-based approach rivals DFT in accuracy and cost-effectiveness. Tested on a large set of reactions, this method was applied to $\mathrm{C}_{4}$ and $\mathrm{C}_{8}$ clusters, and the results approximated coupled-cluster quality at no additional cost.

Reference [34] provided theoretical insights into the structural transformations from polyhedral to spherical fullerenes exposed to radiation and high temperatures. The DFT with tight binding approximation was used to calculate the cross section of electron-induced atomic displacement as a function of the fullerene size. Three types of defects commonly induced by radiation (mono-vacancy, di-vacancy and Stone-Wales defects) were analyzed and their formation energies were determined. The results obtained were consistent with the transformation of polyhedral fullerene structures to spherical nanoonions observed in experiments with electron irradiation. 
In reference [43] a theoretical method that accounts for electron correlation effects and the polarization interaction was used to investigate negative ion formation in lowenergy electron elastic scattering from $\mathrm{C}_{n}(n=24-140)$ fullerenes and the selected heavy lanthanide (Gd and Dy) and actinide ( $\mathrm{Pa}$ and $\mathrm{U}$ ) atoms through the elastic total cross sections calculations. The ground-state anionic binding energies (BEs) for $\mathrm{C}_{n}(n=24,28,44,60,70$, and 82$)$ match excellently the measured electron affinities. It was found that $\mathrm{C}_{74}$ fullerene has the largest anionic groundstate $\mathrm{BE}$ value among the investigated fullerenes and the ground state of the $\mathrm{C}_{140}$ anion has a large $\mathrm{BE}$ as well. These results are expected to inspire further experimental and theoretical explorations of electron attachment in lowenergy electron scattering in these and related systems.

In reference [45] model pseudopotentials for neutral $\mathrm{C}_{60}$ as well as its singly charged positive and negative ions were constructed on the basis of the total electrostatic potentials obtained from DFT calculations. The role of electronic correlations was investigated by comparing the results obtained with different exchange-correlation functionals. The pseudopotentials were fitted by analytical functions to facilitate their use in molecular dynamics simulations.

Structure and properties of polycyclic aromatic hydrocarbons (PAHs) have also been a subject of interest in both experimental and theoretical physics (see, e.g., a recent review [56]). In this Topical Issue, Delaunay et al. [39] studied the interaction of $3 \mathrm{keV} \mathrm{Ar}^{+}$ions with clusters of two structural isomers of $\mathrm{C}_{16} \mathrm{H}_{10}$ PAHs, namely fluoranthene and pyrene. Following the collision with the projectile atom, a rich molecular growth inside the cluster was observed for both isomers. The observed growth processes included the hydrogenation of the parent molecule and the addition of $\mathrm{CH}_{x}$ units.

\section{Molecular-level mechanisms of radiation induced biodamage}

Experimental and theoretical studies of radiation and collision processes with biomolecules and biomolecular clusters as well as numerical simulations of such processes are highly relevant nowadays in connection with the molecular-level assessment of biological damage induced by radiation [48-50]. The scientific interest in obtaining a deeper understanding of radiation damage is motivated by the development of ion-beam cancer therapy [49,51,52] and other applications of ions interacting with biological targets [57].

At present, despite substantial experimental advances there is still a lack in understanding of the mechanism of radiation damage at the molecular level. Therefore, medical protocols have been established mainly on the basis of the experience and practical knowledge of personnel and on empirical methods, rather than on fundamental scientific principles. In recent years, however, it has been shown that a detailed mechanistic understanding of these processes is indeed possible due to new advances in the theoretical and experimental tools developed in atomic, molecular and atomic cluster physics [49,52].
One of impressive examples is the discovery of resonant mechanisms at the origin of radiation damage caused by very low-energy electrons via dissociative electron attachment (DEA) [58]. The identification of such a quantum effect paved the way to new research on possible lowenergy radiation damage effects $[49,50,52]$. It is known that molecular environments can strongly influence DEA processes. Several mechanisms for DEA suppression and enhancement due to cluster environments, particularly due to nano-hydration, were reviewed in reference [26] of this Topical Issue. Long-range electron-molecule and electron-cluster interactions play often a significant role in these effects and can be analyzed by using theoretical models. The review [26] focused mainly on the theoretical description of physical mechanisms underlying numerous experimental observations.

An important issue in studying interactions of ion beams with biomolecular targets is an accurate quantitative description of the primary effects of biodamage due to the incident ions and of the role of secondary particles generated by primary radiation. Several papers published in this Topical Issue explored these effects.

Kitajima et al. [42] analyzed experimentally the formation of secondary ions in microdroplets of liquid methanol bombarded by $0.4-4.0 \mathrm{MeV}$ carbon ions. Positive and negative secondary ions including molecular fragments, methanol clusters and reaction products were investigated, and each ion yield was examined as a function of electronic stopping power $S_{e}$. Different $S_{e}$-dependencies were observed on the emission yields between positive and negative ions. For positive cluster ions $\left[\left(\mathrm{CH}_{3} \mathrm{OH}\right)_{n}+\mathrm{H}\right]^{+}$ $(n=2-10)$, the yield increases non-linearly and follows a power law dependence, $S_{e}^{\alpha}$ with $\alpha=3$. For negative secondary ions, the value of $\alpha$ varies from 0 to 1.5 according to secondary ion species or ion mass.

It was predicted theoretically that one of the possible pathways of ion-induced radiation damage is related to the thermomechanical effect, - a shock wave produced by ions [59]. In reference [38] of this Topical Issue the radial energy deposition around carbon ion paths was analyzed theoretically including the contribution of energetic $\delta$-electrons. The radial profiles of energy deposition and the induced concentrations of free radicals were used to simulate the shock waves by means of reactive classical molecular dynamics. The simulation results predicted a characteristic distribution of reactive chemical species which may have an as yet unrecognised contribution to the nascent biodamage. The paper also suggested some experimental methods by which such a shock wave may be detected experimentally and the predictions of these simulations verified.

In reference [36] a methodology for calculation of survival curves with an account for ion tracks' interference was developed using the multiscale approach to the physics of radiation damage with ions [52]. The method was applied to cells for which shouldered survival curves have been obtained experimentally. It was demonstrated that deviation from the exponential dependence of cell survival probability on deposited dose, due to ion tracks' interference, is observed only at extremely high doses on the order of kGy. At doses relevant for medical 
applications this effect may not be relevant and shouldered cell survival curves arise due to DNA damage repair mechanisms. The recipe was designed for both high and low values of linear energy transfer and can be applied not only for cells but also to plasmid DNA and other biological targets.

The need for a better understanding of the relationship between the physical characteristics of ionizing radiation and the consequent physical, chemical, and biological processes in living matter initiated an extensive research effort on this topic. Nevertheless, many questions about the complex biological effects of ionizing radiation still remain to be answered. An important task is the evaluation of the extent and character of radiation-induced complex DNA lesions as well as understanding of the mechanisms of repair of these lesions. In this regard, the paper by Depes et al. [41] presented recent advances in super-resolution light microscopy, such as the development of single-molecule localization microscopy (SMLM), which offers an optical resolution of approximately an order of magnitude better than that of standard confocal microscopy and thus opens new horizons in radiobiological research. SMLM not only offers a resolution on the order of approximately $10 \mathrm{~nm}$ but provides also entirely new information on the biochemistry and spatio-temporal organization of DNA double strand breaks and their repair at the molecular level. First attempts to use SMLM to explore $\gamma \mathrm{H} 2 \mathrm{AX}$ and 53BP1 repair foci induced with ${ }^{15} \mathrm{~N}$ high-LET particles were presented.

\section{Atomic clusters and complex molecular systems as radiosensitizers}

Besides their scientific attraction, atomic clusters, nanoparticles and complex molecular systems are of increasing practical relevance in physics, chemistry, biology, materials science and medicine. One of hot research topics of atomic and molecular physics and cluster science is related to the development of radiosensitizers, i.e., molecules, clusters or nanoparticles that can enhance radiation effects on biological targets. These systems may act in combination with radiation (e.g., by binding to a specific site of the target biomolecule) and thus can be exploited in radiotherapy or radiochemotherapy to increase effectiveness of treatment of radioresistant tumors. One of actively explored research topics is the investigation of relevant physical and chemical processes behind the proposed enhanced radiation damage in the presence of molecular radiosensitizers and radiosensitizing nanoparticles.

Several papers in this Topical Issue are focused on the properties of molecular radiosensitizers and coated (ligand-protected) nanoparticles. Langer et al. [28] presented a combined experimental and theoretical study of the interaction of electrons with two structurally similar organometallic molecules, $\mathrm{Cp}_{2}^{\star} \mathrm{TiCl}_{2}$ and $\mathrm{Cp}_{2}^{\star} \mathrm{TiF}_{2}\left(\mathrm{Cp}^{\star}=\right.$ $\left.\mathrm{C}_{5}\left(\mathrm{CH}_{3}\right)_{5} \mathrm{H}\right)$, which are of potential interest for concominant chemo-radiotherapy applications. Experimental measurements of partial cross sections for the DEA and the electron ionization mass spectra of isolated molecules were reported. It was demonstrated that the DEA mechanism is rather different in the two molecules: DEA to $\mathrm{Cp}_{2}^{\star} \mathrm{TiF}_{2}$ primarily results in the formation of a stable parent anion, while DEA to $\mathrm{Cp}_{2}^{\star} \mathrm{TiCl}_{2}$ results in the strong fragmentation, primarily into a $\mathrm{Cl}^{-}$anion. Due to the distinct behavior of these molecules for DEA, they were suggested as the model species for studying the role of DEA in postirradiation chemistry.

A combined ion mobility-mass spectrometry approach was used in reference [37] for the analysis of ultrasmall gold nanoclusters protected by glutathione (SG) ligands. SG-coated gold nanoclusters have been suggested as potential radiosensitizers for radiation therapy [60]. Collision cross section (CCS) measurements were reported in [37] for different charge states of $\mathrm{Au}_{10}(\mathrm{SG})_{10}$, $\mathrm{Au}_{11}(\mathrm{SG})_{11}$ and $\mathrm{Au}_{12}(\mathrm{SG})_{12}$. Semi-empirical calculations at the PM7 level of theory were performed to optimize geometrical structures of the clusters and use them to compute CCS. The comparison of the experimentally and theoretically determined CCS allowed to draw conclusions on the structural changes, in particular partial unfolding of SG ligands, upon charging.

Finally, reference [29] presented the results of a theoretical and numerical study of the transport of lowenergy electrons through the coating of a radiosensitizing metallic nanoparticle under fast ion irradiation. The case study represented a poly(ethylene glycol)-coated gold nanoparticle of diameter $1.6 \mathrm{~nm}$ excited by carbon ions of $0.3-10 \mathrm{MeV} / \mathrm{u}$ energy. The diffusion equation for lowenergy electrons emitted from a finite-size spherical source representing the surface of the metal core was solved to obtain the electron number density as a function of radial distance and time. Information on the atomistic structure and composition of the coating was obtained from molecular dynamics simulations. Two mechanisms of lowenergy electron production by the metallic core were considered: the relaxation of plasmon excitations and collective excitations of valence $d$ electrons in individual atoms of gold. Diffusion coefficients and characteristic lifetimes of electrons propagating in gold, water, and poly(ethylene glycol) were obtained from relativistic partial wave analysis and the dielectric formalism, respectively. On this basis, the number of electrons released through the organic coating into the surrounding aqueous medium and the number of hydroxyl radicals produced were evaluated. It was demonstrated that the water content of the coating, especially near the surface of the metal core, is crucial for the production of $\mathrm{OH}$ radicals.

\section{Concluding remarks}

Atomic cluster science has continued its significant progress in recent years, but a large number of questions in the field still remain open. Different types of clusters, various cluster geometries and size ranges, composite and ligand-protected clusters, complex molecules (including biomolecules), clusters on a surface and embedded into nanodroplets or matrices, all provide additional topics which make this field of science very rich and diverse. 
Photon, electron, atom and ion collisions involving atomic clusters, mass spectrometry and laser techniques provide state-of-the-art tools for experimental studies of the cluster structures and properties.

All these questions remain among the key topics of the International Symposia on Atomic Cluster Collisions. The ISACC conference series continues and the next, ninth ISACC conference will take place in Canterbury, United Kingdom from July 31 to August 03, 2019. Similar to previous ISACC conferences, it will focus on the structure and dynamics of atomic clusters and nanoparticles; structure and dynamics of biomolecules; cluster structure and dynamics on a surface; cluster-molecule interactions, reactivity and nanocatalysis; clustering in systems of various degrees of complexity; clusters and biomolecules in external (e.g., electric, magnetic, laser) fields; electron, photon and ion-cluster collisions; collision processes with biomolecules; as well as on cluster and biomolecular research with free electron lasers.

AV is grateful for the financial support from the DKFZ Postdoctoral Fellowship.

\section{References}

1. A.V. Solov'yov, J.-P. Connerade, Latest Advances in Atomic Cluster Collisions: Fission, Fusion, Electron, Ion and Photon Impact (Imperial College Press, 2004)

2. J.-P. Connerade, A.V. Solov'yov, Latest Advances in Atomic Cluster Collisions: Structure and Dynamics from the Nuclear to the Biological Scale (Imperial College Press, 2008)

3. J.A. Alonso, Structure and Properties of Atomic Nanoclusters, 2nd edn. (Imperial College Press, 2012)

4. I.A. Solov'yov, A.V. Solov'yov, W. Greiner, Int. J. Mod. Phys. E 13, 697 (2004)

5. E.E. Campbell, Fullerene Collision Reactions (Springer Science \& Business Media, 2003)

6. R.D. Beck, J. Rockenberger, P. Weis, M.M. Kappes, J. Chem. Phys. 104, 3638 (1996)

7. T. Schlathölter, F. Alvarado, S. Bari, A. Lecointre, R. Hoekstra, V. Bernigaud, B. Manil, J. Rangama, B. Huber, ChemPhysChem 7, 2339 (2006)

8. I.V. Hertel, H. Steger, J. de Vries, B. Weisser, C. Menzel, B. Kamke, W. Kamke, Phys. Rev. Lett. 68, 784 (1992)

9. S.W.J. Scully, E.D. Emmons, M.F. Gharaibeh, R.A. Phaneuf, A.L.D. Kilcoyne, A.S. Schlachter, S. Schippers, A. Müller, H.S. Chakraborty, M.E. Madjet, J.M. Rost, Phys. Rev. Lett. 94, 065503 (2005)

10. A.V. Solov'yov, Int. J. Mod. Phys. B 19, 4143 (2005)

11. A.V. Verkhovtsev, A.V. Korol, A.V. Solov'yov, P. Bolognesi, A. Ruocco, L. Avaldi, J. Phys. B: At., Mol. Opt. Phys. 45, 141002 (2012)

12. H. Haberland, T. Hippler, J. Donges, O. Kostko, M. Schmidt, B. von Issendorff, Phys. Rev. Lett. 94, 035701 (2005)

13. A. Aguado, M.F. Jarrold, Annu. Rev. Phys. Chem. 62, 151 (2011)
14. Z.Y. Li, N.P. Young, M. Di Vece, S. Palomba, R.E. Palmer, A.L. Bleloch, B.C. Curley, R.L. Johnston, J. Jiang, J. Yuan, Nature 451, 46 (2008)

15. D.M. Wells, G. Rossi, R. Ferrando, R.E. Palmer, Nanoscale 7, 6498 (2015)

16. J. Rossbach, J.R. Schneider, W. Wurth, Phys. Rep. 808, 1 (2019)

17. B. Schütte, J. Lahl, T. Oelze, M. Krikunova, M.J.J. Vrakking, A. Rouzée, Eur. Phys. J. D 70, 115 (2016)

18. A.M. Dow, A.R. Wittrig, H.I. Kenttämaa, Eur. J. Mass Spectrom. 18, 77 (2012)

19. T.M. Jarrell, B.C. Owen, J.S. Riedeman, B.M. Prentice, C.J. Pulliam, J. Max, H.I. Kenttämaa, J. Am. Soc. Mass Spectrom. 28, 1091 (2017)

20. O. González-Magaña, M. Tiemens, G. Reitsma, L. Boschman, M. Door, S. Bari, P.O. Lahaie, J.R. Wagner, M.A. Huels, R. Hoekstra, T. Schlathölter, Phys. Rev. A 87, 032702 (2013)

21. A.V. Solov'yov, E. Surdutovich, eds., in The Fourth International Symposium "Atomic Cluster Collisions: Structure and Dynamics from the Nuclear to the Biological Scale" (ISACC 2009), AIP Conf. Proc. (AIP Publishing, 2009), Vol. 1197

22. A.V. Korol, A.V. Solov'yov, Eur. Phys. J. D 67, 1 (2013)

23. R. Prosmiti, P. Villarreal, G. Delgado-Barrio, A.V. Solov'yov, Eur. Phys. J. D 71, 36 (2017)

24. A.V. Solov'yov, J.-P. Connerade, W. Greiner, Phys. Scr. 69, C45 (2004)

25. M. Márquez-Mijares, O. Roncero, P. Villarreal, T. González-Lezana, Eur. Phys. J. D 72, 79 (2018)

26. I.I. Fabrikant, Eur. Phys. J. D 72, 96 (2018)

27. A. Castillo-García, T. González-Lezana, G. DelgadoBarrio, P. Villarreal, Eur. Phys. J. D 72, 102 (2018)

28. J. Langer, M. Zawadzki, M. Fárník, J. Pinkas, J. Fedor, J. Kočišek, Eur. Phys. J. D 72, 112 (2018)

29. K. Haume, P. de Vera, A. Verkhovtsev, E. Surdutovich, N.J. Mason, A.V. Solov'yov, Eur. Phys. J. D 72, 116 (2018)

30. O. Borrell-Grueiro, U. Baños-Rodríguez, M. MárquezMijares, J. Rubayo-Soneira, Eur. Phys. J. D 72, 121 (2018)

31. S. Raggl, N. Gitzl, P. Martini, P. Scheier, O. Echt, Eur. Phys. J. D 72, 130 (2018)

32. F. Calvo, R. Hamdi, L. Mejrissi, B. Oujia, Eur. Phys. J. D 72, 133 (2018)

33. A.J.C. Varandas, Eur. Phys. J. D 72, 134 (2018) [Erratum: Eur. Phys. J. D 72, 180 (2018)]

34. R.E. Sosa-Ricardo, D. Codorniu-Pujals, M. MárquezMijares, Eur. Phys. J. D 72, 137 (2018)

35. S.M. Ciborowski, G. Liu, J.D. Graham, A.M. Buytendyk, K.H. Bowen, Eur. Phys. J. D 72, 139 (2018)

36. E. Surdutovich, A.V. Solov'yov, Eur. Phys. J. D 72, 140 (2018)

37. C. Comby-Zerbino, F. Bertorelle, F. Chirot, Ph. Dugourd, R. Antoine, Eur. Phys. J. D 72, 144 (2018)

38. P. de Vera, E. Surdutovich, N.J. Mason, F.J. Currell, A.V. Solov'yov, Eur. Phys. J. D 72, 147 (2018)

39. R. Delaunay, A. Mika, A. Domaracka, B.A. Huber, P. Rousseau, Eur. Phys. J. D 72, 149 (2018)

40. S. König, M. Wolfram, S. Bandelow, G. Marx, L. Schweikhard, Eur. Phys. J. D 72, 153 (2018) 
41. D. Depes, J.-H. Lee, E. Bobkova, L. Jezkova, I. Falkova, F. Bestvater, E. Pagacova, O. Kopecna, M. Zadneprianetc, A. Bacikova, E. Kulikova, E. Smirnova, T. Bulanova, A. Boreyko, E. Krasavin, M. Hausmann, M. Falk, Eur. Phys. J. D 72, 158 (2018)

42. K. Kitajima, H. Tsuchida, T. Majima, M. Saito, Eur. Phys. J. D 72, 169 (2018)

43. A.Z. Msezane, Z. Felfli, Eur. Phys. J. D 72, 173 (2018)

44. L. Savio-Rodríguez, A. Gutiérrez-Quintanilla, G. RojasLorenzo, P. Pajón-Suárez, J. Rubayo-Soneira, Eur. Phys. J. D 72, 195 (2018)

45. K.B. Agapev, I.I. Vrubel, R.G. Polozkov, V.K. Ivanov, Eur. Phys. J. D 72, 196 (2018)

46. A.C. Reber, S.N. Khanna, Eur. Phys. J. D 72, 199 (2018)

47. X. Wu, A. Alvarez-Ibarra, D.R. Salahub, A. de la Lande, Eur. Phys. J. D 72, 206 (2018)

48. I. Baccarelli, F.A. Gianturco, E. Scifoni, A.V. Solov'yov, E. Surdutovich, Eur. Phys. J. D 60, 1 (2010)

49. A.V. Solov'yov, Nanoscale Insights into Ion-beam Cancer Therapy (Springer International Publishing, Cham, Switzerland, 2017)

50. G. García Gómez-Tejedor, M.C. Fuss, Radiation Damage in Biomolecular Systems (Springer Science+Business Media B.V., Dordrecht, 2012)
51. D. Schardt, T. Elsässer, D. Schulz-Ertner, Rev. Mod. Phys. 82, 383 (2010)

52. E. Surdutovich, A.V. Solov'yov, Eur. Phys. J. D 68, 353 (2014)

53. K. Haume, S. Rosa, S. Grellet, M.A. Śmiałek, K.T. Butterworth, A.V. Solov'yov, K.M. Prise, J. Golding, N.J. Mason, Cancer Nanotechnol. 7, 8 (2016)

54. G. Song, L. Cheng, Y. Chao, K. Yang, Z. Liu, Adv. Mater. 29, 1700996 (2017)

55. S. König, A. Jankowski, G. Marx, L. Schweikhard, M. Wolfram, Phys. Rev. Lett. 120, 163001 (2018)

56. M. Gatchell, H. Zettergren, J. Phys. B: At., Mol. Opt. Phys. 49, 162001 (2016)

57. M. Durante, F.A. Cucinotta, Rev. Mod. Phys. 83, 1245 (2011)

58. B. Boudaïffa, P. Cloutier, D. Hunting, M.A. Huels, L. Sanche, Science 287, 1658 (2000)

59. E. Surdutovich, A.V. Solov'yov, Phys. Rev. E 82, 051915 (2010)

60. X.-D. Zhang, Z. Luo, J. Chen, S. Song, X. Yuan, X. Shen, H. Wang, Y. Sun, K. Gao, L. Zhang, S. Fan, D.T. Leong, M. Guo, J. Xie, Sci. Rep. 5, 8669 (2015) 